\title{
Persée
}

http://www.persee.fr

\section{Entre production et institutionnalisation des idées. La réforme de la Politique agricole commune}

\author{
Ève Fouilleux
}

Revue française de science politique, Année 2000, Volume 50, Numéro 2

p. 277 - 306

\section{Voir l'article en ligne}

La grille d'analyse proposée dans cet article apour objectif de comprendre l'articulation entre les phénomènes de production et d'institutionnalisation des idées. Elle associe une approche institutionnaliste des instruments de politique publique au concept de forum, opérationnalisé à travers la sociologie de la traduction et la notion d'échange politique. La partie empirique étudie les processus qui ont mené à la réforme de la Politique agricole commune de 1992. Elle analyse la production par I'OCDE de nouveaux critères de bonne politique agricole domes- tique dans les années 1980 à partir d'idées issues du forum scientifique des économistes, puis leur introduction par la Commission dans la négociation inter gouvernementale européenne. Le rôle d'encadrement de la production des idées par les instruments de politique publique est ensuite souligné à travers l'évolution des débats scientifique et professionnel comme consé-quence du changement de 1992.

\section{Avertissement}

L'éditeur du site «PERSEE » - le Ministère de la jeunesse, de l'éducation nationale et de la recherche, Direction de l'enseignement supérieur, Sous-direction des bibliothèques et de la documentation - détient la propriété intellectuelle et les droits d'exploitation. A ce titre il est titulaire des droits d'auteur et du droit sui generis du producteur de bases de données sur ce site conformément à la loi n`98-536 du 1 er juillet 1998 relative aux bases de données.

Les oeuvres reproduites sur le site «PERSEE » sont protégées par les dispositions générales du Code de la propriété intellectuelle.

Droits et devoirs des utilisateurs

Pour un usage strictement privé, la simple reproduction du contenu de ce site est libre.

Pour un usage scientifique ou pédagogique, à des fins de recherches, d'enseignement ou de communication excluant toute exploitation commerciale, la reproduction et la communication au public du contenu de ce site sont autorisées, sous réserve que celles-ci servent d'illustration, ne soient pas substantielles et ne soient pas expressément limitées (plans ou photographies). La mention Le Ministère de la jeunesse, de l'éducation nationale et de la recherche, Direction de l'enseignement supérieur, Sous-direction des bibliothèques et de la documentation sur chaque reproduction tirée du site est obligatoire ainsi que le nom de la revue et- lorsqu'ils sont indiqués - le nom de l'auteur et la référence du document reproduit.

Toute autre reproduction ou communication au public, intégrale ou substantielle du contenu de ce site, par quelque procédé que ce soit, de l'éditeur original de l'oeuvre, de l'auteur et de ses ayants droit.

La reproduction et l'exploitation des photographies et des plans, y compris à des fins commerciales, doivent être autorisés par l'éditeur du site, Le Ministère de la jeunesse, de l'éducation nationale et de la recherche, Direction de l'enseignement supérieur, Sous-direction des bibliothèques et de la documentation (voir http://www.sup.adc.education.fr/bib/ ). La source et les crédits devront toujours être mentionnés. 


\title{
ENTRE PRODUCTION \\ ET INSTITUTIONNALISATION DES IDÉES : \\ LA RÉFORME DE LA POLITIQUE AGRICOLE COMMUNE
}

\author{
ÈVE FOUILLEUX
}

$\mathrm{D}$

e nombreux travaux mettent l'accent sur le rôle des idées, des représentations, des valeurs dans les politiques publiques. Néanmoins, la plupart les abordent comme des données a priori, non comme l'objet central de la recherche. Les idées sont considérées soit comme des inputs du processus décisionnel, celles-ci façonnant finalement les politiques produites ${ }^{1}$, soit comme des sous-produits du processus de décision, les politiques constituant des « sources d'information et de sens » pour le grand public ${ }^{2}$. Mais la question des modalités de la production des idées et de leur émergence dans le débat comme recettes d'action publique n'est jamais directement posée. C'est ce point aveugle de l'articulation entre la production des idées, leur prise en compte comme alternatives envisageables et leur institutionnalisation sous la forme d'instruments de politique publique que nous proposons d'explorer dans cet article.

\section{POUR UNE APPROCHE FORMALISÉE DU RÔLE DES IDÉES DANS LES POLITIQUES PUBLIQUES}

\section{LES IDÉES COMME VARIABLES EXPLICATIVES DU CHANGEMENT INSTITUTIONNEL}

En tant qu'ensemble d'instruments organisant des transactions entre les acteurs (en l'occurrence entre les pouvoirs publics et les agriculteurs) une politique publique est un ensemble de règles du jeu. Si l'on assimile les règles du jeu à des institutions ${ }^{3}$, une politique publique est donc également un ensemble d'institutions.

Quoique fortement imbriquées et dépendantes les unes des autres, toutes les institutions d'une politique ne peuvent être étudiées en même temps. Nous distinguerons

1. Cf. les différentes contributions de l'ouvrage de A. Faure, G. Pollet, P. Warin, La construction du sens dans les politiques publiques. Débats autour de la notion de référentiel, Paris, L'Harmattan, 1995. Dans la littérature néo-institutionnaliste anglo-saxonne, cf. P. A. Hall (ed.), The Political Power of Economic Ideas : Keynesianism across Nations, Princeton, Princeton University Press, 1989 et « Policy Paradigms, Social Learning and the State. The case of Economic Policymaking in Britain », Comparative Politics, 25 (3), 1993, p. 275-296. Cf. également P. A. Sabatier, H. C. Jenkins-Smith, Policy Change and Learning. An Advocacy Coalition Approach, Boulder, Westview Press, 1993.

2. Cf. P. Pierson, «When Effect Becomes Cause. Policy Feedback and Political Change », World politics, 45, 1993, p. 595 628.

3. Cf. D. C. North, Institutions, Institutional Change and Economic Performance, Cambridge, Cambridge University Press, 1990, ainsi que «Institutions », Journal of Economic Perspectives, 5 (1), 1991, p. 97-112. 
donc les institutions endogènes à l'analyse (en l'occurrence les instruments de la PAC), dont il s'agira d'expliquer les variations, des institutions exogènes, qui assurent le cadre général de l'interaction. Les attributions et compétences des différents acteurs du système politique européen et les règles du processus décisionnel de la PAC par exemple, stables pendant la période étudiée, peuvent être considérées comme exogènes.

L'analyse consistera à expliquer le changement des institutions endogènes de la PAC à partir de la variable des idées, c'est-à-dire comme un changement d'idées institutionnalisées, dont il s'agira de retracer le processus progressif d'émergence, de sélection et d'institutionnalisation. L'approche dominante d'analyse des politiques agricoles est celle de l'économie politique, qui considère les «intérêts » comme variable explicative principale de l'inertie ou du changement institutionnel '. Sans renier l'apport de ces travaux, nous adopterons dans ce papier une perspective volontairement différente, en nous intéressant exclusivement aux articulations et interactions existant entre idées et institutions.

Notons, par ailleurs, qu'il s'agisse des « idées », des « intérêts » ou des « institutions », ces variables n'ont de pertinence réelle à nos yeux qu'à travers leur intériorisation, leur revendication plus ou moins explicite par différents acteurs. Centrer l'analyse sur ces acteurs nous permettra d'éviter le piège fonctionnaliste et le manque d'applicabilité empirique qui peuvent découler de l'utilisation du schéma des trois variables en « $i$ ». Les idées seront donc entendues comme désignant un ensemble de représentations, de grilles d'analyse et de schémas d'entendements divers, qui font sens à travers leur incarnation dans des communautés d'acteurs spécifiques (les intérêts seront ainsi pris en compte, à travers les représentations que s'en font les acteurs concernés).

\section{LES ENJEUX POLITIQUES DE LA PRODUCTION DES IDÉES}

\section{Des forums et des idées}

Plusieurs auteurs ont utilisé ou repris la notion de « forum » dans leurs travaux. Ils leur donnent des définitions plus ou moins proches, plus ou moins précises, les assimilant soit à des réseaux ou des communautés, soit à des organisations particulières, soit à des lieux ou des regroupements d'acteurs spécifiques. En partant des travaux de Bruno Jobert ${ }^{2}$, nous définirons les forums comme des scènes plus ou moins institutionnalisées, régies par des règles et des dynamiques spécifiques, au sein desquels des acteurs ont des débats touchant de près ou de loin à la politique publique que l'on étudie. Chaque forum est producteur de représentations, «d'idées » sur la politique, qui peuvent être interprétées par référence à l'identité, aux intérêts des acteurs qui

1. Cf., par exemple, H. Guyomard, L. P. Mahé, K. Munk, T. Roe, «L'agriculture au GATT et la réforme de la PAC: l'éclairage de l'économie politique et de l'économie publique », Économie rurale, 220-221, 1994, p. 175-180. Cette approche est assez décevante dans l'explication de la réforme de la PAC de 1992 par rapport aux précédentes, basées sur des systèmes de contingentement de l'offre. Si, comme ces dernières, elle a conservé à l'identique les effets redistributifs budgétaire et financiers de la politique, elle a néanmoins fondamentalement bouleversé la «philosophie » de ses instruments.

2. B. Jobert, Le tournant néolibéral en Europe, Paris, L'Harmattan, 1994. Cf. également du même auteur, «Représentations sociales, controverses et débats dans la conduite des politiques publiques », Revue française de science politique, 42 (2), avril 1992, p. 219-234. 
l'habitent et aux rapports de force qui les opposent, ainsi que, plus fondamentalement, au type de règles du jeu qui le régissent (institutions formelles et informelles ').

Les forums permettent de rendre intelligibles l'hétérogénéité des idées existantes autour d'une politique publique et la pluralité des systèmes de représentation et d'action dans lesquelles ces idées s'inscrivent. Les critères d'acceptabilité et de cohérence du discours ne sont pas les mêmes d'un forum à l'autre ; les controverses n'y évoluant ni parallèlement ni au même rythme, les dissonances peuvent être fortes entre eux. Leurs règles du jeu respectives étant spécifiques, les idées produites et manipulées sur l'un ou l'autre à propos d'une même politique peuvent être de nature très différente, voire complètement antagonistes. Comme nous le verrons dans la suite, en permettant d'éclairer les différentes provenances possibles des idées institutionnalisées, l'utilisation de l'outil « forum » dans une perspective dynamique permet également de rendre intelligibles certains paradoxes ou aberrations inhérents à une politique ou à son référentiel.

Pour cela, nous distinguons deux grandes catégories de forums : ceux assurant une activité de production d'idées sur les politiques, d'une part (forums scientifique, professionnel, de la rhétorique politique), et le forum des communautés de politique publique, d'autre part, lieu de réutilisation, d'institutionnalisation des idées à travers leur transformation en instruments de politique publique. Sur la base de cette distinction, nous supposons qu'il existe une circulation d'idées des forums de production d'idées vers le forum des communautés de politique publique, dont il s'agit de saisir les étapes successives et les modalités. Conformément à notre choix initial de souligner le caractère « incarné » des idées et des mécanismes de leur diffusion, une telle perspective impliquera d'analyser les échanges et déplacements d'acteurs en jeu entre les différents forums.

\section{Processus traductionnels, porte-parole et sélection des alternatives}

Dans notre définition, les forums de production des ides sont des lieux de débats plus ou moins spécialisés au sein desquels s'affrontent plusieurs groupes d'acteurs développant des stratégies pour faire prévaloir leur vision du monde. Pour étudier le cas des politiques agricoles, nous avons considéré trois forums de production des idées :

- Le forum de l'économie scientifique est régi par la recherche de l'excellence à l'intérieur du paradigme dominant et les règles de l'argumentation sont guidées en son sein par l'impératif de rigueur scientifique du raisonnement. Le forum scientifique des économistes est occupé par des spécialistes de diverses questions. Celui qui nous intéresse est le forum des économistes agricoles. Il est à la fois soumis aux impératifs théoriques généraux de la science économique et aux enjeux propres à une discipline « appliquée » au domaine de la production agricole.

- Sur le forum de la communication politique (de la thétorique politique), l'enjeu central est la conquête et/ou la conservation du pouvoir politique, qui passe par la construction de coalitions, la dévalorisation des adversaires et l'argumentation, en vue de convaincre les électeurs. La production « d'idées » sur ce forum prend donc essen-

1. D. C. North (Institutions..., op. cit.) distingue les institutions «formelles » (constitutions, lois, règlements) des institutions «informelles» (normes, coutumes, conventions, croyances, idéologies). 
tiellement la forme de programmes et de discours électoraux. Son activité générale est rythmée par les différentes échéances électorales qui ponctuent la vie politique.

- Le forum professionnel ne figure pas dans la définition des forums proposée initialement par Bruno Jobert, les acteurs de la société civile n'intervenant dans son approche que sur le forum des communautés de politique publique. Dans notre approche, le forum professionnel est le lieu d'une production d'idées sur la politique agricole, à partir de la défense de modèles spécifiques (idéaux) de pratique de l'agriculture. Divers groupes d'agriculteurs (éventuellement organisés en mouvements syndicaux) s'y affrontent et tentent de faire valoir, tant du côté de leur «base » que du côté des pouvoirs publics, leur vision de ce que doit être l'agriculture. Chacun des modèles d'agriculture en question est le dépositaire d'une identité agricole spécifique, dont découle une définition de « l'intérêt agricole » à défendre '.

Ces forums ne sont pas mobilisés au même moment dans la régulation. Tandis que le forum professionnel, sectoriel et spécialisé, produit en permanence des représentations sur la politique agricole, le forum de la rhétorique politique ne va cristalliser son discours sur des questions agricoles qu'à certains moments critiques (élections induisant la nécessité de mobilisation de la population agricole et rurale par exemple). Le forum scientifique n'est pas consacré dans sa totalité à la politique agricole, autour de laquelle n'est rassemblée qu'une petite fraction de spécialistes « sectoriels », mais son activité de production d'idées se fait en continu et sur long terme.

En revanche, ces scènes ont en commun d'être les lieux d'une lutte pour la domination ayant pour enjeu l'imposition d'un ensemble d'idées, de représentations spécifiques comme « référent central ». Non seulement le référent central du forum encadre les débats et assure une stabilité relative des échanges, mais il permet aux acteurs de se situer : qu'ils s'y conforment ou qu'ils le refusent, leur positionnement par rapport au référent dominant est un critère identitaire majeur. Résultant de constructions sociales différentes, les référents centraux de chaque type de forum ne peuvent être considérés comme étant de nature équivalente sur le plan analytique : le référent central du forum des économistes scientifiques est de type " paradigmatique ${ }^{2}$, le référent central orientant les échanges sur la scène de la rhétorique politique est de type « doctrinaire », ou «idéologique », tandis que sur la scène professionnelle, nous préférerons le terme de «modèle » d'agriculture ${ }^{3}$. Nous reviendrons sur ce point dans les paragraphes consacrés au référentiel, référent du forum des communautés de politiques publiques.

Sur le plan cognitif, le référent central peut être considéré comme l'institution centrale du forum, « institution informelle » dans la typologie de D. C. North ${ }^{4}$. Condi-

1. Comme modèles antagonistes d'agriculture en concurrence sur le forum, on peut citer, par exemple l'agriculture conventionnelle (dite "moderne»), intensive et spécialisée, d'une part, et l'agriculture biologique (et ses variantes, comme l'agriculture biodynamique), refusant notamment tous les intrants chimiques, d'autre part. Cf. J.-P. Revéret, J. Peltier, H. Boudier, «De l'agriculture conventionnelle à l'agriculture écologique, vers un nouveau paradigme », Sociologies et sociétés, 13, 1983, p. 49-62.

2. En l'appliquant au seul forum scientifique, nous restreignons ici volontairement le terme de paradigme à son sens kuhnien originel. Cf. T. S. Kuhn, La structure des révolutions scientifiques, Paris, Flammarion, 1983, ou encore La tension essentielle. Tradition et changement dans les sciences, Paris, Gallimard, 1990.

3. Nous préférerons la notion de «modèle d'agriculture» à celle de «modèle professionnel ». Au sens où nous l'entendons, tout modèle d'agriculture inclut dans sa définition un modèle professionnel spécifique, mais il comprend également un rapport plus explicite au territoire, à la nature et au type de techniques agricoles employées.

4. Cf. D. C. North, Institutions..., op. cit. 
tionnant la production intellectuelle de l'ensemble du forum, la définition d'une telle institution est un enjeu de pouvoir majeur. En ce sens, l'émergence d'un référent dominant correspond à une première étape de sélection des alternatives de politique publique, au terme d'un processus que nous proposons de formaliser à travers les outils de la sociologie de la traduction proposés par Michel Callon '. L'objectif de la sociologie de la traduction est d'explorer les mécanismes de surgissement, développement et clôture des controverses scientifiques à travers l'émergence d'un groupe d'acteur comme porte-parole d'une communauté sur un sujet donné. Le répertoire de la traduction se décline en quatre étapes successives : la problématisation, l'intéressement, l'enrôlement, la mobilisation. Il se termine au moment où la traduction devient trahison, à travers le phénomène de dissidence, qui provoque l'enclenchement d'une nouvelle controverse.

Nous supposerons ainsi que la dynamique interne respective de chacun des forums de production des idées est de type traductionnel : elle se concrétise simultanément par l'imposition d'un référent dominant et par la désignation du groupe d'acteurs qui s'en réclame comme porte-parole légitime du forum. L'une des dimensions fondamentales du pouvoir conféré au porte-parole est d'émettre sa propre vision du monde à l'extérieur du forum, au nom du forum tout entier : « Parler pour d'autres, c'est d'abord faire taire ceux au nom desquels on parle ${ }^{2}$. Le processus de traduction conférant aux groupes victorieux une forte visibilité et une forte légitimité, ils deviennent naturellement les interlocuteurs privilégiés pour l'extérieur du forum. L'activité traductionnelle est ainsi au centre du mécanisme de production et de visibilisation des idées, tant à l'intérieur qu'à l'extérieur du forum.

L'émergence d'un référent donné en tant que référent central, c'est-à-dire reconnu comme tel par la majorité des acteurs du forum, n'empêche pas pour autant l'existence continue de voix dissidentes qui le refusent. L'équilibre de domination d'un référent et de ses défenseurs est toujours précaire, susceptible d'être remis en cause à un autre moment par l'entrée en dissidence majoritaire des acteurs concernés. Ceci peut se produire lors d'une nouvelle élection sur le forum de la rhétorique politique, lors d'une élection syndicale sur le forum professionnel, lors d'une « révolution scientifique ${ }^{3}$ sur le forum des économistes. En fait, la controverse n'est jamais vraiment close. Elle peut néanmoins être considérée comme telle sur un forum donné, si les acteurs dissidents restent marginaux et n'ont pas la capacité (les ressources politiques) d'induire une mise en cause réelle du référent dominant.

Sur le forum professionnel, par exemple, le processus traductionnel des jeunes agriculteurs jacistes au cours de leur « révolution silencieuse ${ }^{4}$ dans les années 1950 leur a non seulement permis de s'ériger en porte-parole du monde agricole à travers l'investissement des structures syndicales unitaires ${ }^{5}$, mais également d'imposer leur

1. M. Callon, «Éléments pour une sociologie de la traduction. La domestication des coquilles Saint-Jacques et des marins-pêcheurs dans la baie de Saint-Brieuc », L'Année sociologique, 36, 1986, p. 169-208.

2. Ibid., p. 196.

3. Cf. T. S. Kuhn, La structure des révolutions scientifiques, op. cit.

4. Cf. M. Debatisse, La révolution silencieuse. Le combat des paysans, Paris, CalmannLévy, 1963.

5. Au terme de sa victoire traductionnelle, le groupe initialement « informel » des représentants des jeunes agriculteurs a «institutionnalisé » son statut de porte-parole à travers l'investissement des structures syndicales (Centre national des jeunes agriculteurs, puis Fédération nationale des syndicats d'exploitants agricoles), ce qui lui a permis de protéger sa position hégémonique sur le forum bien au-delà de la stricte phase de victoire traductionnelle. 
vision de l'agriculture «moderne » à l'ensemble du forum. Pendant de longues années, le modèle moderniste' fut considéré comme le seul modèle légitime de développement par les agriculteurs français, et la rhétorique professionnelle dominante a complètement occulté les modèles agricoles alternatifs théorisés et promus par quelques agriculteurs marginaux. C'est ainsi que l'agriculture biologique, tout comme l'agriculture « économe-autonome » en Bretagne, par exemple, sont restées complètement inconnues tant à l'intérieur qu'à l'extérieur du forum, alors qu'elles étaient mises en œuvre avec succès sur le terrain depuis de longues années ${ }^{2}$. Elles n'ont par conséquent jamais été envisagées comme des alternatives viables de développement économique agricole (comme nous y reviendrons dans la dernière partie de cet article, la controverse s'est modifiée sur ce point ces toutes dernières années).

Mettant l'accent sur la dimension politique de la production des idées à travers l'acquisition du statut de porte-parole, la sociologie de la traduction permet ainsi de dépasser un simple usage métaphorique de la notion de forum et d'en faire un outil analytique plus opérationnel et plus dynamique. Elle montre que le processus de sélection des alternatives envisageables pour la politique publique commence «à la source », bien en amont de leur mise sur l'agenda politique et de leur apparition dans le débat public : le processus traductionnel de production des idées en est la première étape.

\section{CIRCULATION DES IDÉES, ALIMENTATION DE LA CONTROVERSE ET STRUCTURATION DES RAPPORTS DE FORCE}

Sur le forum des communautés de politique publique a lieu une autre étape de sélection des idées, plus souvent étudiée dans la littérature, qui correspond à leur institutionnalisation à travers la fabrication de la politique. L'enjeu consiste maintenant à comprendre pourquoi et comment une idée donnée se retrouve sur ce dernier forum et y est plus ou moins envisagée comme une alternative possible pour la politique, puis pourquoi et comment elle est ensuite institutionnalisée (transformation en instrument) ou pas.

\section{Traduction, échanges politiques et importation des idées}

Dans sa composition, le forum des communautés de politique publique est caractérisé par une forte hétérogénéité des acteurs en présence, ce qui le distingue fortement des forums de production des idées, dont la population est homogène. On y trouve :

1. Cf. P. Muller, Le technocrate et le paysan, Paris, Les Éditions ouvrières, 1984, ainsi que C. Servolin, L'agriculture moderne, Paris, Le Seuil, 1989. Le modèle moderniste est centré sur des valeurs de progrès, de professionnalisme. Du point de vue agronomique et technico-économique, il est basé sur une intensification des facteurs de production et caractérisé par l'association investissement/ agrandissement/ spécialisation.

2. Pour une bonne synthèse concernant l'agriculture biologique, cf. C. de Silguy, L'agriculture biologique, Paris, PUF, 1997. Concernant les expériences bretonnes d'agriculture « économe-autonome » et le système du " tout à l'herbe », cf. André Pochon, La prairie temporaire à base de trêfle blanc, Plérin, CEDAPA, début des années 1980 et Du champ à la source, Plérin, CEDAPA, 1991. 
- Des responsables politiques élus tout d'abord, susceptibles d'être remplacés à chaque élection. Porte-parole du forum de la rhétorique politique, ils deviennent les « décideurs publics » sur le forum des communautés de politique publique, ce qui leur confère une place particulière : à la fois détenteurs de la violence légitime et « responsables » devant les citoyens, ils affirment leur volonté de rester au pouvoir qui conditionne directement la dynamique générale du forum ;

- Des responsables administratifs ensuite, moins directement soumis aux aléas électoraux. Les élites administratives ont un rôle primordial à la fois dans la continuité de l'action publique et dans la fabrication du compromis : en charge des opérations de décodage-recodage du réel nécessaires à l'élaboration de la politique ', ce sont des acteurs-clés dans l'importation, les recombinaisons et l'agglomération des idées qui servent à la fabrication des instruments de politique publique ;

- Des acteurs provenant des autres forums enfin : leaders professionnels et autres représentants de la société civile, experts issus du forum scientifique. Ce sont généralement les porte-parole des forums de production des idées (comme nous l'avons souligné, un des enjeux majeurs de la conquête d'un tel statut réside dans l'accès qu'il procure au forum des communautés de politique publique).

Ces différents acteurs participent au débat concernant la politique publique à produire. Leur influence est fonction des ressources dont ils disposent pour imposer leurs idées. La dynamique générale du forum des communautés de politique publique n'est pas de type traductionnel comme dans les cas précédents, mais orientée vers la poursuite de l'échange politique et le maintien du compromis. La sociologie de la traduction éclaire néanmoins certains éléments de cette dynamique car elle est directement conditionnée par les transactions établies avec les forums de production des idées : la qualité de la traduction sur les premiers est directement dépendante de la nature des relations établies avec les acteurs du forum des communautés de politique publique (mise en place de dispositifs d'intéressement et d'enrôlement pour l'obtention du statut de porte-parole). Mais cette dépendance forte des acteurs des forums de production des idées vis-à-vis de ceux du forum des communautés de politique publique est souvent réciproque, les seconds pouvant nécessiter leur appui pour être légitimés dans leur action de décideurs publics ou être directement « demandeurs » des idées produites par les premiers pour mener à bien cette action.

Ces liens sont ainsi à l'origine de la mise en place d'échanges politiques entre les différents forums, vecteurs potentiels du passage des idées des uns aux autres (même si ces dernières ne sont pas forcément l'objet officiel de l'échange) : ils assurent l'alimentation des débats et controverses qui servent de substrat à la fabrication et aux modifications éventuelles des recettes et instruments de politique publique. Le graphique 1 illustre la nature de ces échanges.

C'est ainsi que le débat des politiques publiques est amené à reprendre des concepts et des notions formulés ailleurs, dans des contextes différents, qu'il adapte, dégrade, hybride. En effet, le passage d'une idée d'un forum à l'autre ne se fait jamais sans une certaine déformation. Cela découle de notre définition initiale des forums comme scènes institutionnalisées, c'est-à-dire régulées par des institutions spécifiques : impliquant obligatoirement une adaptation de l'idée aux règles du jeu de son

1. Cf. B. Jobert, P. Muller, L'État en action. Politiques publiques et corporatismes, Paris, PUF, 1987, ou encore P. Muller, Les politiques publiques, Paris, PUF, 1990. 
forum d'adoption (forcément différentes de celles de son forum d'origine), toute activité d'importation/exportation inter-forums induira automatiquement une déformation plus ou moins marginale de celle-ci ${ }^{~}$.

Graphique 1. Échanges politiques et interactions entre forums

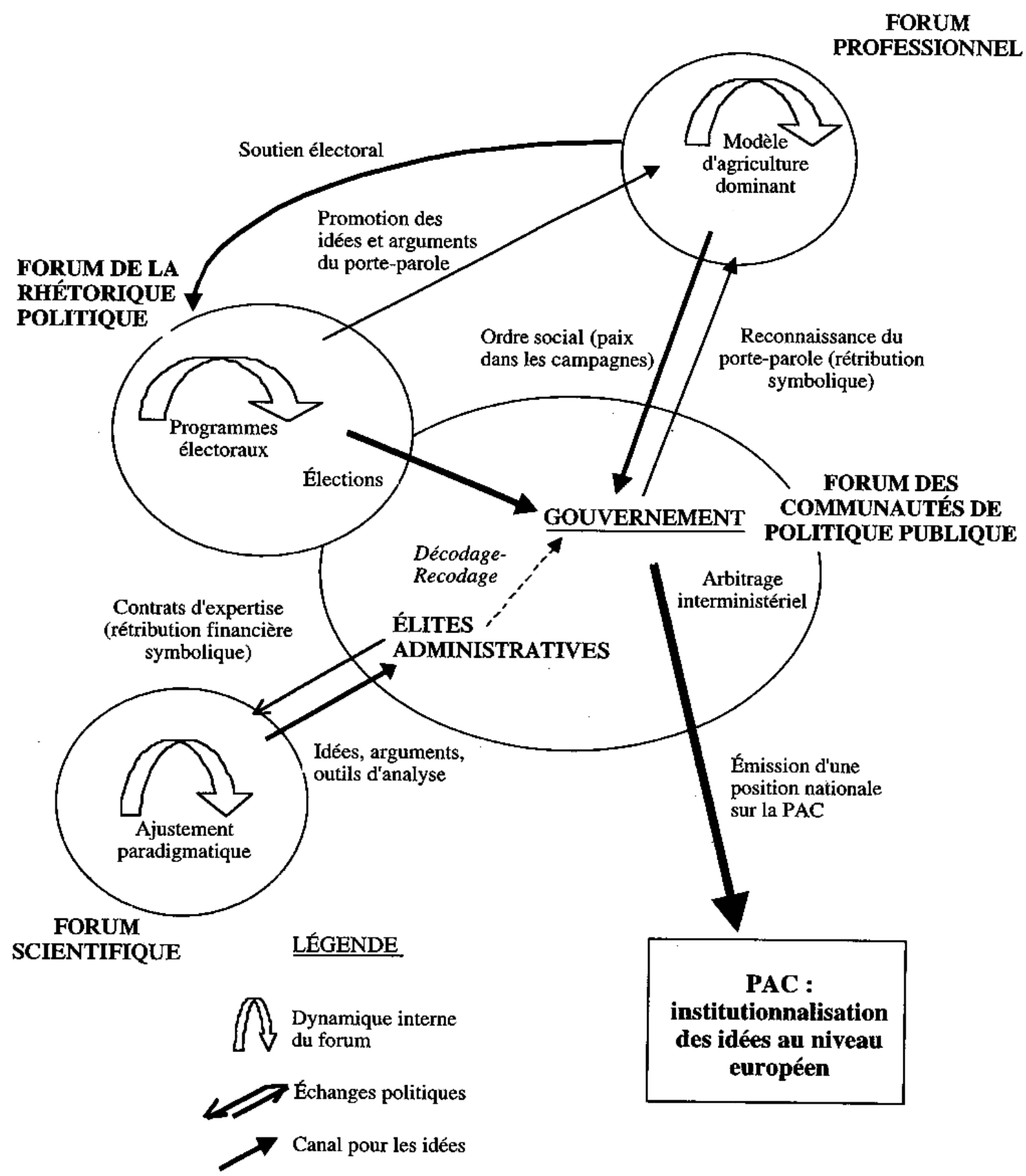

1. Ainsi, par exemple, les économistes scientifiques déplorent souvent la façon avec laquelle sont repris leur langage et leurs concepts dans le débat politique, l'utilisation de leurs travaux consistant souvent à reprendre les seuls résultats des recherches, sans mention des hypothèses qui, dans leur logique scientifique, balisent strictement le domaine de validation des résultats, dont elles sont même un élément à part entière. 
L'échange qui lie le forum des communautés de politique publique à celui de la rhétorique politique est hautement institutionnalisé. À travers le mécanisme général de l'élection, les vainqueurs du processus de traduction sur le forum de la rhétorique politique se retrouvent à incarner le « décideur public ». L'échange établi entre les deux forums permet donc l'accès du parti ou de la coalition partisane dominante au gouvernement, qui bénéficie alors de la légitimité démocratique par les urnes. Les idées contenues dans les programmes électoraux sont ainsi automatiquement importées sur le forum des communautés de politique publique, mais elles sont loin d'être automatiquement institutionnalisées pour autant, les règles du jeu régissant leur nouveau forum d'adoption (maintien du compromis) étant bien différentes de celles qui ont permis à leurs défenseurs d'obtenir leur victoire (argumentation et thétorique politique).

Au niveau national, l'échange politique établi entre le forum des communautés de politique publique et le porte-parole professionnel a été analysé dans le domaine agricole comme un échange de type néocorporatiste ' . Le porte-parole du forum professionnel assure une certaine stabilité de l'ordre public à travers le «maintien de la paix dans les campagnes », contre quoi le gouvernement, représentant l'État, assure un soutien financier au secteur à travers les politiques redistributives mises en œuvre et une légitimation du statut de porte-parole à travers sa sélection comme interlocuteur syndical unique. Si l'échange principal a lieu entre les responsables agricoles et le gouvernement, autrement dit les hommes politiques élus, les différents travaux sur la cogestion ont montré qu'il se répercutait également avec les élites administratives sectorielles, qui nourrissent des relations suivies avec «la profession» (syndicats, chambres d'agriculture, etc.) ${ }^{2}$.

Les échanges susceptibles de s'établir entre le forum des communautés de politiques publiques et le forum scientifique prennent essentiellement la forme de demandes d'expertise par les pouvoirs publics, qui fournissent en retour une rétribution financière et symbolique aux scientifiques concernés, utile sur leur forum d'origine; les activités d'expertise sont également l'occasion pour les acteurs du forum scientifique d'avoir accès à des données (statistiques en particulier) dont la diffusion est parfois restreinte. L'échange établi entre le forum scientifique et le forum des communautés de politique publique passe aussi par le choix des conseillers du gouvernement (cabinets), qui fournit aux acteurs concernés un accès « aux sphères du pouvoir ", qui, outre les ressources symboliques qu'il procure peut leur être directement utile d'un point de vue professionnel (recrutements, dispositifs de financement de la recherche, par exemple). Comme nous l'illustrerons dans la partie empirique de cet article, la qualité du passage des idées d'un forum à l'autre dans le cadre de cet échange est assez intimement liée à l'orientation intellectuelle des élites administratives impliquées. Des facteurs structurels et culturels propres aux administrations impliquées (différences nationales ou sectorielles) sont susceptibles de conditionner l'intensité des échanges.

1. P. Muller, B. Jobert, L'État en action..., op. cit.

2. Cf. notamment P. Coulomb, H. Delorme, B. Hervieu, M. Jollivet, P. Lacombe, Les agriculteurs et la politique, Paris, Presses de Sciences Po, 1990 : cf. en particulier la revue des travaux sur la cogestion dans le chapitre de J.-P. Billaud. Cf. aussi C. Servolin, L'agriculture moderne, op cit. 


\section{Union européenne : à système polycentrique, échanges politiques multiples}

Qu'elle soit qualifiée de système politique polycentrique, de multi-level governance ' ou de composite polity ${ }^{2}$, l'Union européenne présente des singularités qui la distinguent du modèle de l'État-nation ${ }^{3}$, qui a implicitement servi de base à la description de notre cadre analytique. Ce n'est pas l'objet de ce papier que de développer dans le détail notre propre approche du système politique européen, mais il convient néanmoins de donner quelques indications quant aux particularités de la circulation des idées dans un tel cadre. Dans un système polycentrique comme l'Union européenne, les différents pôles de décision sont en effet à l'origine d'enjeux de légitimation et d'échanges politiques intra et inter-forums démultipliés par rapport à un système national.

Compte tenu des attributions des différents acteurs du système politique européen et des règles du processus de décision de la PAC, le débat agricole européen est alimenté en idées par deux voies formelles principales: les États membres et la Commission ${ }^{4}$.

- Les premiers sont représentés au sein du (des) Conseil(s) des ministres à travers leurs positions nationales. La position d'un État à Bruxelles peut être considérée comme le résultat d'une première institutionnalisation d'idées provenant du débat du forum national des communautés de politique publique. La participation des États au débat européen renvoie ainsi à la configuration évoquée dans les paragraphes précédents de cet article, c'est-à-dire aux forums et aux échanges politiques nationaux.

- À travers son pouvoir de proposition, la Commission joue un rôle important dans le débat. Ses interfaces avec les forums de production des idées sont essentiellement la mobilisation d'expertise sur le forum scientifique, d'une part, et la consultation des professionnels agricoles, $d$ 'autre part, que celle-ci passe par le COPA, porteparole «européen » officiel du forum professionnel, ou par diverses formes de lobbying particularistes auprès de ses services. La partie empirique montrera par ailleurs que son interface avec le niveau international du forum a eu une importance particulière en 1991-1992.

Comme nous l'avons développé ailleurs ${ }^{5}$, les changements d'une politique sectorielle européenne peuvent ainsi être interprétés comme des ajustements de type

1. Cf. R. Putnam, « Diplomacy and Domestic Politics : The Logic of Two-Level Games », International Organization, 42, 1988, p. 427-460. Pour une approche critique, cf. A. Smith, «Studying Multi-Level Governance. Examples from French Translations of the Structural Funds », Public Administration, 75 (4), hiver 1996.

2. D. Imig, S. Tarrow, "Studying Contention in an Emerging Polity », dans D. Imig, S. Tarrow (eds), Contentious Europeans. Politics and Protest in a Composite Polity, Lanham, Rowman Littlefield (à paraître en 2000).

3. Pour une synthèse de la littérature, cf., par exemple, C. Lequesne, A. Smith, « Union européenne et science politique : où en est le débat théorique ? », Cultures et Conflits, 28, 1997, p. 7-31.

4. Si un certain nombre d'autres acteurs sont également susceptibles d'intervenir dans le débat (Parlement européen, Cour de justice européenne), leurs faibles prérogatives concernant la PAC au cours de la période étudiée nous autorisent à les laisser de côté dans le cadre de ce papier.

5. È. Fouilleux, « Changement de politique publique dans l'Union européenne : la Politique agricole commune entre permanences et innovations », Politiques et management public, $15(1), 1997$, p. 117-137. 
global/sectoriel, impliquant les États par l'intermédiaire du Conseil européen (dépositaire des enjeux globaux) et du Conseil de l'agriculture (dépositaire des enjeux sectoriels) et la Commission européenne comme médiateur de l'ajustement. En charge du décodage-recodage du réel au niveau communautaire à travers ses propositions au Conseil, le rôle de la Commission serait ainsi analogue à celui des élites administratives au niveau national, mais « compliqué » par son statut au sein du système politique européen. Disposant de ressources institutionnelles et politiques propres, elle est susceptible de mettre en œuvre des stratégies pour accroître son pouvoir et sa légitimité (comportements d'《entrepreneur politique »). De ce point de vue, elle est désavantagée par rapport aux gouvernements nationaux car la forte visibilité que lui confere son rôle de proposition (forte imputabilité des réformes) n'est pas compensée par l'existence d'un forum de la rhétorique politique où elle puisse justifier son action (ni au niveau communautaire ni dans les États membres).

L'inexistence d'un réel forum européen de la rhétorique politique explique sans doute en partie que la Commission ait dû endosser le rôle de bouc émissaire dans ces réformes, les gouvernements nationaux se « déchargeant » sur elle de leur responsabilité dans les décisions politiques. Il semble qu'elle essaie aujourd'hui de promouvoir des politiques qui la mettent moins facilement en difficulté en même temps qu'elles lui confèrent un pouvoir plus important (politiques régulatrices). Elle développe par ailleurs des dispositifs propres de légitimation, notamment à travers ses campagnes de promotion de l'intégration européenne et la production de dispositifs symboliques.'.

\section{STABILITÉ, CRISE ET CHANGEMENT DE POLITIQUE PUBLIQUE}

\section{Arène, forum : deux configurations des débats de politique publique}

Vecteurs éventuels de l'importation des idées, les échanges politiques établis entre le forum des communautés de politique publique et les autres forums conditionnent également son activité de production institutionnelle. À travers les rapports de force qu'ils engendrent et en fonction des ressources détenues par les différents acteurs impliqués, la déstabilisation éventuelle de ces échanges peut directement menacer le compromis établi sur ce dernier. Ils sont ainsi susceptibles d'avoir des conséquences importantes sur l'état du forum lui-même (stabilité - crises), ainsi que sur l'évolution de la politique publique concernée (inertie ou changement).

Comme on l'a déjà évoqué, les règles du jeu du forum des communautés de politique publique correspondent essentiellement à la conservation du compromis et à la poursuite de l'échange politique, c'est-à-dire à la recherche d'un mode de définition de la réalité sociale qui rend possible l'obtention d'un compromis entre les différentes parties prenantes sur la politique publique à mettre en œuvre. Quand ces règles du jeu sont enfreintes, autrement dit lorsque le compromis politique est directement et ouvertement menacé (défection d'un acteur disposant de ressources importantes dans l'échange, par exemple), le forum change de configuration : il entre en phase instable, de renégociation du compromis, que l'on qualifiera de conjoncture critique. Ce terme nous semble effectivement préférable à celui de « crise », trop définitif, partiel, sub-

1. Cf. C. Lager, L'Europe en quête de ses symboles, Euroclio: Études et documents, Berlin, Lang, 1995. Sur une facette spécifique du métier politique européen, cf. aussi A. Smith, «Des élites sans territoires : les commissaires européens », Pôle Sud, 7, 1997. 
jectif et péjorativement connoté '. Invisibles en phase stable bien qu'ils existent en continu pour définir les termes de l'échange entre les acteurs ${ }^{2}$, les débats sont rendus publics en phase de conjoncture critique : sans pour autant lui être immédiatement et facilement intelligibles, ils deviennent visibles pour le grand public.

La «vie » du forum des communautés de politique publique est ainsi rythmée par l'alternance de phases de stabilité et de conjonctures critiques qui permet de repérer deux types de dynamiques des idées :

- une configuration « forum » correspondant à une phase de mise en veille de la négociation, à la maturation progressive de la controverse et à une nouvelle étape de sélection des alternatives issues des différents forums de production des idées ;

- une configuration «arène », conjoncture critique du forum, étape ultime de la sélection des alternatives qui se clôt à travers le renouvellement du compromis. Ce moment de négociation/délibération est caractérisé par une forte visibilisation des débats.

L'arène offrant le spectacle de l'aboutissement du processus progressif de maturation et de sélection des alternatives sur le forum, le passage d'une configuration à l'autre se caractérise par une forte réduction du nombre d'alternatives en discussion. C'est ainsi au moment où le nombre d'idées à discuter est le plus réduit que le débat s'ouvre au « grand public » ${ }^{3}$.

La conjoncture critique nous intéresse particulièrement car elle est susceptible de se clore par l'institutionnalisation de certaines des idées en débat. Une telle issue n'est évidemment pas systématique. Pour être institutionnalisée, une idée doit non seulement être importée par un partenaire disposant de ressources dans l'échange, mais également permettre d'assurer la conservation ou le renouvellement du compromis

1. Cf. sur ce point l'introduction de B. Jobert, Le tournant néolibéral en Europe, op. cit. et J. Lagroye, « La légitimation », dans M. Grawitz, J. Leca (eds), La science politique science sociale. L'ordre politique, Paris, PUF, 1985, p. 395-467. Cf. également J. P. Burgess, « Legitimacy between Institution and Identity. What's European about the European Union? », Florence, Institut universitaire européen, Centre Robert Schuman, Seminar paper EUR/22, 10 février 2000.

2. Le témoignage suivant de l'économiste Joseph Klatzmann à propos des réunions de la Commission de terminologie de l'économie agricole au ministère français de l'Agriculture, auxquelles il participait en tant qu'expert, illustre tout à la fois l'impossible clôture de la controverse en phase stable, et le caractère néanmoins essentiel de l'élaboration d'un langage propre au forum : «En plusieurs années de travail, nous avons en tout et pour tout présenté une cinquantaine de définitions, la plupart sous la forme suivante : un texte court, suivi de plusieurs remarques. Lorsqu'une définition était proposée par l'un d'entre nous, nous en discutions longuement, remettant à peu près toujours la suite de la discussion à la réunion suivante. Et lorsqu'une définition était adoptée, il est arrivé fréquemment qu'elle soit remise en cause plus tard. Nous avons ainsi "remis sur le tapis" certaines définitions jusqu'à neuf fois. Et la décision finale a souvent été prise à la majorité, dans l'impossibilité de réaliser l'unanimité. J'ai dit une fois au cours d'une réunion "Cela fait bientôt cinquante ans que je parle de l'agriculture et je me rends compte aujourd'hui que je ne sais pas vraiment ce que c'est" (étant donné l'impossibilité d'en définir les frontières) » (J. Klatzmann, "Ce qui m'a le plus frappé », Économie rurale, 200, 1990, p. 16).

3. En évoquant « l'espace de négociation » à propos des arènes et « l'espace des débats » pour les forums, Bruno Jobert distingue ces deux notions d'un point de vue spatial (cf. B. Jobert, "Rhétorique politique, controverses scientifiques et construction des normes institutionnelles : esquisse d'un parcours de recherche », dans A. Faure et al., La construction du sens..., op. cit., p. 19). La différenciation que nous faisons ici pour notre part entre forum et arène est plutôt d'ordre temporel. 
concernant la politique. Si elle n'alimente pas directement cette dynamique, l'idée aura toutes les chances de rester en suspens dans le débat.

Cependant, du fait de la forte visibilisation qui caractérise la conjoncture critique et des enjeux de légitimation politique qui lui sont liés, si une idée bénéficie d'une audience favorable auprès du grand public, par exemple, ne remplissant pas directement ces conditions, elle pourra servir de légitimation rhétorique à des changements d'instruments n'ayant aucun lien direct avec elle (ce qui peut constituer un premier pas vers une institutionnalisation future). Autrement dit, elle pourra être invoquée pour justifier l'institutionnalisation d'idées complètement distinctes (voire antagonistes).

Par ailleurs, si l'idée est introduite par un partenaire influent et que son institutionnalisation ne remet pas en cause l'économie générale du compromis, elle pourra être intégrée à la politique sous la forme de dispositifs marginaux (mesures non obligatoires sur le plan légal ou peu dotées du point de vue budgétaire, par exemple), qui pourront également être brandis comme des éléments de légitimation d'un changement plus radical. L'institutionnalisation marginale de ces nouvelles idées poussées par un partenaire influent de l'échange peut également servir au moment de la négociation du compromis global à infléchir un peu la position de celui-ci dans d'autres domaines (situation de négociation « inflationniste », classique au niveau communautạire).

\section{Référentiel et verrouillages cognitifs des politiques}

L'activité fondamentale du forum des communautés de politique publique, consiste dans la production de ses propres institutions, dont en particulier la politique publique elle-même. Le "référentiel » (qui désigne ici à la fois les instruments de la politique et leur schéma d'organisation général) peut ainsi être considéré comme un « référent central » un peu particulier sur ce forum. Son caractère hybride, d'une part, et l'étendue de son influence, d'autre part, le distinguent des référents centraux des forums de production des idées.

Il convient tout d'abord de souligner le caractère intrinsèquement hybride du référentiel : par construction, les idées qui le constituent sont issues des différents forums de production des idées, autrement dit de systèmes de représentations présentant des cohérences spécifiques et variées. Une telle approche nous amène ainsi à adopter une position tout à fait tranchée dans la querelle terminologique visant à circonscrire la question du sens dans les politiques publiques : le référent central de la politique publique est défini comme résultant d'une controverse qui emprunte et réutilise des idées issues de débats encadrés par des référents de nature différente, «paradigme scientifique», «modèle professionnel» (identité et techniques), « doctrine » ( «idéologie », « philosophie politique ») notamment. L'utilisation d'un de ces derniers termes pour le désigner occulterait ses autres dimensions originelles, et ôterait à l'analyse les moyens de saisir le caractère fondamentalement hybride qui le caractérise. Parler de "paradigme d'une politique publique " ${ }^{1}$, nous semble ainsi susceptible d'engendrer des confusions préjudiciables à l'analyse, le risque étant d'assimiler le paradigme scientifique (ensemble cohérent et rationnel par définition) à

1. C'est ce que font plusieurs auteurs. Cf., par exemple, P. A. Hall, «Policy Paradigms... », art. cité, F. X. Merrien, "Les politiques publiques entre paradigmes et controverses », dans CRESAL (dir.), Raisons de l'action publique, Paris, L'Harmattan, 1993, p. 87100 , et Y. Surel, « Les politiques publiques comme paradigmes », dans A. Faure et al., La construction du sens..., op. cit. p. 125-151. 


\section{Ève Fouilleux}

la politique publique elle-même (par définition pleine de contradictions du fait de sa diversité génétique et des compromis qu'elle institutionnalise). De la même façon, on évitera de parler de modèle de politique agricole '. Par ailleurs, on évitera de remplacer le terme de «modèle d'agriculture », réservé au forum professionnel par celui de «paradigme d'agriculture » ${ }^{2}$.

Plutôt que de supposer a priori une improbable cohérence à la politique, notre approche du référentiel comme ensemble d'idées institutionnalisées provenant de différents forums de production d'idées permet au contraire d'avancer vers l'explication de l'hétérogénéité et des contradictions internes susceptibles de s'y trouver, tout en rendant « lisible » (car « déconstructible ») cette complexité.

La deuxième spécificité du référentiel réside dans l'étendue de son influence. Institution centrale du forum des communautés de politiques publiques, il encadre directement les échanges entre les acteurs et la nature des débats qui s'y déroulent. Susceptible d'influer sur les chances relatives d'institutionnalisation des différentes idées en débat, il conditionne directement les possibilités de changement envisageables pour la politique. Il peut ainsi être le point d'ancrage cognitif de phénomènes d'inertie et de verrouillages institutionnels et politiques responsables des difficultés à changer la politique. Mais le pouvoir d'encadrement du référentiel s'étend bien au-delà du seul balisage du débat sur le forum des communautés de politique publique : le référentiel est susceptible d'influencer directement les échanges internes aux autres forums.

Qu'il s'agisse de leurs institutions formelles (règles de fonctionnement des établissements d'enseignement et de recherche sur le forum scientifique, statuts de la FNSEA ou du droit rural sur le forum professionnel) ou informelles (nature du référent dominant sur chacun d'entre eux), le rôle d'encadrement des règles du jeu d'un forum de production des idées s'arrête à ses frontières. En revanche, en s'intégrant aux dispositifs institutionnels de l'ensemble des autres forums dont ils deviennent des règles du jeu à part entière, les instruments de la politique permettent au référentiel de s'ajouter à leurs propres institutions et il est alors susceptible d'influencer directement la production d'idées dont ils sont le siège ${ }^{3}$. Le référentiel et les instruments d'une politique sont ainsi susceptibles de réduire le nombre des alternatives envisageables « à la source » du processus, sur les forums de production des idées.

1. L'utilisation de cette notion de «modèle de politique agricole » est fréquente chez des auteurs comme Pierre Coulomb ou Claude Servolin, dans leurs travaux sur les relations État/ profession et le poids du syndicalisme agricole dans la définition de la politique agricole ; ils ont implicitement tendance à assimiler analytiquement le modèle professionnel et le référentiel de la politique agricole. Inversement, il semble parfois que Pierre Muller assimile le référentiel de la politique agricole au modèle professionnel, quand il fait référence à ses travaux sur les lois de modernisation française de 1960-1962 (cf., par exemple, P. Muller, «Les politiques publiques comme construction d'un rapport au monde ", dans A. Faure et al., La construction du sens..., op. cit., p. 153-179).

2. À partir d'une approche agronomique des modèles mis en œuvre, certains auteurs parlent en effet de « paradigmes » d'agriculture (J.-P. Revéret et al., « De l'agriculture conventionnelle à l'agriculture écologique... », art. cité).

3. Une telle influence du référentiel jusque sur les forums de production des idées peut être rapprochée des effets de feed-back évoqués par Paul Pierson («When Effect Becomes Cause... », art. cité), qui considère qu'en tant que « puissants paquets d'incitations et de ressources », les politiques peuvent avoir des conséquences sur les positions des groupes d'intérêt, des élites gouvernementales et des acteurs sociaux individuels ; elles sont notamment des « sources d'information et de sens » pour le grand public, les interprétations politiques auxquelles elles donnent lieu étant notamment fonction du degré d'intelligibilité de leurs instruments. 


\section{ENTRE CRITÈRES INTERNATIONAUX \\ ET VOLONTÉ POLITIQUE EUROPÉENNE : VERS UNE RÉFORME RADICALE DE LA PAC}

Les grandes lignes de notre cadre d'analyse étant établies, l'enjeu consiste maintenant à l'utiliser pour l'étude d'un cas empirique : les transformations de la Politique agricole commune. Il s'agira donc de comprendre les modalités d'alimentation du débat sur la PAC, la sélection progressive des alternatives et la construction des conjonctures critiques, dont, en particulier, celle qui a donné lieu à sa réforme de 1992.

\section{PAC : DE L'INERTIE À LA RÉFORME DE 1992}

Principalement vouée à l'unification et à l'organisătion du marché européen des grands produits agricoles (céréales, viande bovine, lait, etc.), la Politique agricole commune a été prévue dès 1957 dans le traité de Rome (article 39). Elle était principalement basée sur la mise en place de « prix garantis » (parfois aussi désignés comme «prix institutionnels »), pour les principaux produits agricoles, associés à des instruments de protection aux frontières sous la forme de prélèvements variables (droits de douanes et restitutions). Ces instruments conféraient à la PAC un caractère particulièrement « incitatif » à la production !

Assurés de débouchés « au prix fort » quel que soit l'état du marché du fait de la garantie des prix, les producteurs étaient incités à produire en quantité croissante et illimitée. Si toutefois l'offre d'un produit donné était amenée à dépasser la demande, des dispositifs conjoncturels d'intervention publique sur le marché (retrait de quantités appropriées et stockage) étaient destinés à rétablir l'équilibre entre offre et demande. Dès les années 1970, la PAC a ainsi permis d'atteindre l'autosuffisance pour les principaux produits alimentaires. Cependant, l'offre ayant rapidement excédé structurellement la demande de produits agricoles, les stocks ont crû de plus en plus.

Malgré l'accumulation des excédents et l'explosion des dépenses (frais d'achat et de stockage, revente à perte sur le marché mondial), résultats d'autant de dysfonctionnements de la politique, la PAC n'a pas été modifiée. Seuls quelques changements basés sur des dispositifs de limitation quantitative de l'offre (quotas laitiers en 1984, quantités maximales garanties en 1987/1988) ont progressivement été introduits. Mais, d'ampleur systématiquement restreinte et de mise en œuvre peu stricte, ces réformes n'ont pu résoudre les problèmes structurels de la politique.

Pourtant, les scientifiques spécialistes des marchés agricoles dénonçaient depuis l'origine les instruments de la PAC, qu'ils jugeaient inefficaces du fait de leur caractère protectionniste (responsable de distorsions de concurrences sur le marché mondial des produits agricoles) et de l'iniquité des transferts sociaux qu'ils engendraient (proportionnel aux quantités produites, le soutien était majoritairement orienté vers les

1. Si ces instruments de politique économique s'inscrivaient dans une certaine continuité historique avec ceux existant précédemment dans les différents États membres, ils étaient également cohérents avec le contexte social et politique européen de l'époque (pénuries alimentaires consécutives à la seconde guerre mondiale, tensions internationales croissantes liées à la guerre froide) : atteindre l'indépendance alimentaire était un enjeu majeur pour la Communauté européenne. Pour un bon aperçu historique, cf. M. Tracy, L'État et l'agriculture en Europe occidentale. Crises et réponses au cours d'un siècle, Paris, Economica, 1986. 
agriculteurs les plus « gros »). Inscrits dans le paradigme de l'économie du bien-être, ces économistes militaient en faveur d'une élimination (à défaut, d'une baisse) des prix garantis afin de déconnecter l'intervention publique des mécanismes du marché. Pourtant publiquement répétées ', leurs prises de parole n'ont eu aucun effet visible sur le cours de la politique. Les réformes des années 1980, basées sur des systèmes de contrôle de l'offre, étaient même aux antipodes de leurs préconisations. La PAC était tout simplement réputée « impossible à réformer » 2 .

C'est pourtant sur le modèle proposé par les économistes scientifiques qu'a finalement été produite la « Nouvelle PAC » de 1992, basée sur une baisse de $30 \%$ du niveau des prix garantis des grandes cultures et de $15 \%$ pour la viande bovine. Ces baisses de prix sont « compensées » par des aides directes aux producteurs calculées individuellement de façon à procurer à chacun la même quantité de soutien public que l'assuraient les prix garantis (multiplication de la surface cultivée par un rendement de référence historique).

Comment comprendre un tel bouleversement des règles du jeu de la PAC ? Pourquoi, alors que toute réforme radicale était réputée impossible depuis plus de trente années, a-t-elle finalement eu lieu en 1992 ? Pourquoi les prix garantis ont-ils été remplacés par des aides directes, et non par d'autres types d'instruments, comme des quotas, solution jusqu' alors privilégiée par les pouvoirs publics européens ? Comment expliquer que l'idée du découplage du soutien à l'agriculture, défendue en vain depuis de longues années par les économistes scientifiques, a soudain été présentée comme la panacée par les responsables politiques en 1992 ? Le cadre d'analyse présenté plus haut devrait nous permettre de répondre à ces interrogations.

\section{DE L'ÉLABORATION À L'INSTITUTIONNALISATION \\ DE NOUVEAUX CRITĖRES INTERNATIONAUX DE POLITIQUE AGRICOLE}

\section{OCDE : nouveaux problèmes, nouveaux outils, nouvelles recettes}

Devant la dégradation de la situation des marchés internationaux des produits agricoles qui a caractérisé la fin des années 1970 et le début des années 1980, dans un contexte international tendu entre pays membres ${ }^{3}$, l'OCDE a fait émerger une ques-

1. Au niveau national, on peut citer, par exemple, l'article de Louis $\mathrm{P}$. Mahé (économiste à l'INRA de Rennes) et M. Drouet (pseudonyme de Michel Roudet, alors économiste à la direction de la Prévision du ministère des Finances) dans Le Monde du 23 février 1982 : «Une politique protectionniste n'est pas la solution pour l'agriculture française » (p. 20). Au niveau européen, on peut citer «The Siena Memorandum on the Reform of the Common Agricultural Policy », appel à une réforme radicale de la PAC signé par treize économistes scientifiques issus des différents États membres, rendu public à l'université de Sienne le 18 février 1984 dans le cadre d'une conférence de presse.

2. De nombreux travaux d'économie politique ont expliqué «l'impossibilité » de réformer la PAC. Cf., par exemple, B. Gardner, « The Common Agricultural Policy : The Political Obstacle to Reform », The Political Quarterly, 58, 1987, p. 167-179; D. Bergmann, P. Baudin, Politiques d'avenir pour l'Europe agricole, Paris, INRA Economica, 1989 ; G. C. Rausser, D. A. Irwin, « The Political Economy of Agricultural Policy Reform », European Review of Agricultural Economics, 15, 1989, p. 349-366; H. W. Moyer, T. E. Josling, Agricultural Policy Reform. Politics and Process in the EC and USA, Ames, Iowa University Press, 1990.

3. L'activité exportatrice de la Communauté européenne sur les marchés internationaux était perçue par les Etats-Unis comme une menace de leurs parts de marché, qui connaissaient des difficultés de plus en plus grandes pour écouler leur production. 
tion nouvelle sur le forum international des communautés de politique publique. Le « mandat ministériel sur les échanges » signé des 24 ministres du Commerce des pays membres demandait au secrétariat de l'organisation d'examiner les relations entre politiques agricoles et commerce et suggérer des approches pour mieux intégrer l'agriculture au système commercial multilatéral '.

Le problème général était celui de la remise en ordre du commerce international des produits agricoles. Le mandat posait à la fois la question des effets possibles d'une réduction des mesures protectionnistes sur les échanges et la question des effets des politiques agricoles domestiques sur le commerce. La première partie de ce problème n'était pas nouvelle sur la scène internationale, où il était à peu près établi et partagé par les acteurs que l'existence de mesures protectionnistes aux frontières était une entrave aux échanges. En revanche, la seconde partie du problème était beaucoup plus nouvelle : elle supposait un lien entre l'état du commerce mondial et les politiques agricoles domestiques, c'est-à-dire mises en œuvre à l'intérieur des pays. Ce lien n'était ni immédiat ni évident dans le débat à ce moment-là.

Le mandat souhaitait aboutir à des préconisations visant à limiter les effets des politiques domestiques sur le commerce, mais son objectif premier était tout d'abord d'évaluer ces effets. II visait à trouver une méthode d'évaluation qui tienne compte de la grande variété des instruments de politique agricole mis en œuvre pour les différents produits dans les différents pays membres ${ }^{2}$. C'est à ce problème technique qu'ont dû se confronter les économistes du département des Affaires économiques et statistiques du secrétariat de l'OCDE : la nouveauté de la question posait le problème des outils nécessaires à l'élaboration de la réponse. Après un moment de flottement et d'hésitations ${ }^{3}$, c'est finalement grâce aux avancées de la théorie économique du bienêtre, paradigme dominant sur le forum scientifique, et aux techniques de modélisation afférentes qu'ils ont pu résoudre le problème.

Ils se sont d'abord appuyés sur les travaux d'un chercheur américain en économie agricole, Tim Josling qui avait mis au point une méthode d'évaluation du soutien public à l'agriculture basée sur les concepts d'Équivalent subvention à la production (ESP), et d'Équivalent subvention à la consommation (ESC). Ce choix méthodologique et théorique s'est prolongé à travers des collaborations avec plusieurs scienti-

1. Le mandat demandait : «(I) Une analyse des approches et des méthodes permettant de réduire de manière équilibrée et graduelle la protection accordée à l'agriculture et de mieux intégrer celle-ci dans le système commercial multilatéral ouvert, tout en tenant compte des caractéristiques et du rôle spécifiques de l'agriculture... ; (II) Un examen des politiques et des mesures nationales en cause qui ont des effets significatifs sur les échanges agricoles en vue d'aider les responsables de la politique dans l'élaboration et la mise en ouvre des politiques agricoles ; (III) Une analyse des méthodes les plus appropriées pour améliorer le fonctionnement du marché agricole mondial. Cette étude pourrait prendre pour point de départ les résultats qu'ont donné plusieurs dispositifs - bilatéraux et multilatéraux - et chercher à déterminer les meilleures approches possibles pour l'avenir». Cf. OCDE, Politiques nationales et échanges agricoles, Paris, OCDE, 1987.

2. Si la Communauté européenne intervenait directement sur ses marchés à travers les prix garantis, les États-Unis, par exemple, soutenaient activement leur agriculture, mais sous la forme d'aides variables versées directement aux producteurs (deficiency payments). Les systèmes de politique agricole des autres pays membres de l'OCDE étaient encore différents.

3. Ces acteurs ont tout d'abord pensé reprendre la méthodologie dite des coefficients de protection effective et nominale (NPC et EPC), déjà utilisée par le gouvernement australien dans le domaine industriel, mais qui s'est avérée inutilisable du fait des spécificités du secteur agricole. 
fiques spécialisés dans le domaine des politiques agricoles et du commerce international '.

Premièrement, les calculs d'ESP/ESC ont permis une mesure et une estimation des composantes de l'aide à l'agriculture, produit par produit, dans les différents pays de l'OCDE ${ }^{2}$. Deuxièmement, la mise au point d'un modèle économique, baptisé « modèle MTM », visant à exploiter les données issues des calculs d'ESP/ESC, permettait de disposer d'un instrument cohérent d'analyse quantitative d'évaluation des incidences d'une réduction progressive ("graduelle et équilibrée ») de la protection accordée à l'agriculture ${ }^{3}$.

Si ces outils et ces calculs font aujourd'hui partie des exercices de routine à l'OCDE, il faut souligner l'innovation majeure qu'ils ont constituée au départ :

« Il s'agissait là de quelque chose de radicalement nouveau par rapport à tout ce qu'avait fait l'OCDE avant, vous savez. Parler d'élasticités de l'offre et de la demande, on n'avait jamais fait ce genre de trucs à l'OCDE. C'est comme le fait de dériver des modèles pour représenter les choses : on n'avait vraiment jamais fait ça avant ! C'était donc une affaire de changement radical, et ça a pris longtemps pour que les gens digèrent, absorbent tout ça et s'y embarquent » (un acteur du secrétariat général).

\section{Vers une première institutionnalisation}

Basés sur les calculs d'ESP, les travaux de l'OCDE ont induit et diffusé un changement complet de problématisation de la politique agricole. La pertinence d'une politique domestique n'était plus regardée par rapport à ses effets sur le revenu des agriculteurs ou à un quelconque paramètre lié à l'activité agricole elle-même ou à l'économie interne du pays concerné comme cela était traditionnellement le cas, mais au regard d'un critère chiffré unique, relatif à ses effets sur le commerce international.

1. Outre les Américains Josling et Pearson, on peut citer notamment le professeur allemand Stephan Tangermann et le Français Louis Mahé de l'INRA de Rennes (par ailleurs auteurs de nombreux articles favorables à une réforme radicale de la PAC et signataires du Memorandum de Sienne).

2. Dans la définition de l'OCDE, l'ESP était conçu comme une mesure unique du soutien, incluant toutes les mesures de soutien des prix (prix garantis, prix d'intervention, gestion de l'offre), les subventions aux intrants (engrais, investissements et crédits), les aides directes (soutien des revenus, paiements compensatoires, deficiency payments), et les aides d'ordre général (soutien à la recherche, à l'agrandissement des exploitations, à la formation, compensations aux catastrophes naturelles, etc.). Cet ESP était exprimé soit en valeur absolue, soit en pourcentage de la production agricole finale, soit par unité de produit. Il permettait des comparaisons des mesures de soutien entre produits, entre pays et dans le temps. Cf. « Modélisation des conséquences des politiques agricoles », Revue économique de l'OCDE, 13, 1990 et aussi A. Buckwell, « Agricultural Economics in a Brave Liberal World », dans European Association of Agricultural Economists (ed.), Redefining the Roles for European Agriculture, Edinburgh, EAAE, 1996, p. 1-17.

3. Le modèle MTM (Trade Mandate Model) donne une représentation de l'économie agricole mondiale pour les productions animales et végétales. Basé sur une série de 11 modèles de structure similaire par pays ou par région dans lesquels des relations endogènes expliquent les facteurs économiques déterminant l'offre, la demande et les prix pour 18 catégories de produits agricoles, reliés entre eux par des échanges nets, il permet de mettre en lumière l'interdépendance des pays et des produits dans l'économie agricole mondiale. Il s'agit d'un modèle de statistique comparative à moyen terme dont l'objectif est d'estimer, au terme d'une période d'ajustement de 5 ans, l'incidence des modifications des politiques agricoles sans rendre compte du sentier d'ajustement suivi pendant cette période (OCDE, « Modélisation... », art. cité, p. 48-49). 
Cette façon nouvelle d'appréhender les politiques agricoles a nourri des phénomènes d'apprentissage d'une ampleur considérable sur le forum :

« Au fil du temps s'est mis en place un véritable processus d'éducation des policy makers, parce que vraiment en agriculture, vingt ans en arrière, je ne crois pas qu'il soit exagéré de dire qu'ils étaient plutôt carrément illettrés en termes de compréhension du fonctionnement des politiques et de leur impact. Les gens n'en avaient aucune idée... Dans ce contexte, le travail fait à l'OCDE a constitué un projet éducatif majeur; il a vraiment permis de sensibiliser un grand nombre de personnes. Il y a quinze ans vous auriez parlé de l'impact d'une politique sur le commerce, les gens vous auraient regardé comme si vous étiez fou ! Un impact sur le commerce? Mais qu'est-ce que vous voulez dire par là ? Ca n'a aucun impact! Quoi, une distorsion commerciale ? Comment cela peut-il se faire ? Vraiment ça en était à ce niveau-là ! Maintenant ça ne fait absolument plus aucun doute pour personne : évidemment que ça a un impact sur le commerce, on le sait...».

Outre la diffusion de connaissances nouvelles (« les politiques agricoles ont un impact sur le commerce $»$ ), les travaux de l'OCDE ont été instrumentalisés dans le débat politique au niveau international du forum : ils ont fourni aux pays favorables à une négociation multilatérale sur l'agriculture au GATT (les États-Unis en particulier) des arguments incontournables dans ce sens. Ayant directement participé à l'élaboration des travaux, donc implicitement reconnu leur validité, les pays défavorables à une telle décision (l'Union européenne en particulier) n'avaient plus d'arguments pour s'y opposer. C'est ainsi qu'ils ont abouti à une première institutionnalisation à travers l'ouverture d'un volet de négociation spécifiquement agricole dans le cadre de l'Uruguay Round du GATT en 1986.

Ainsi, non seulement la déclaration ministérielle d'inauguration de l'Uruguay Round abandonnait la référence au statut « spécial » dont l'agriculture avait bénéficié jusqu'alors (autorisant des exceptions aux règles générales du traité), mais, dans la continuité des réflexions de l'OCDE, elle posait également la question de la réforme des politiques domestiques :

« Les négociations devront avoir pour but d'obtenir une plus grande libéralisation du commerce en agriculture et de ramener toutes les mesures affectant l'accès à l'importation et la concurrence aux exportations dans les règles et les disciplines du GATT... une amélioration de l'environnement de la concurrence passant par une discipline accrue dans l'utilisation de toutes les subventions directes ou indirectes et les autres mesures affectant directement ou indirectement le commerce agricole, ceci incluant de réduire par étapes leurs effets négatifs et de s'occuper de leurs causes $\gg$ '.

Comme le montrera la suite, cette décision sans précédent depuis l'origine du traité en 1948 a eu des répercussions cruciales : le passage des discussions de l'OCDE, basées sur un échange avec le forum scientifique, et dont l'enjeu était de se mettre d'accord sur des outils communs d'analyse, à une négociation consistant à établir les règles du jeu du commerce international des produits agricoles, a constitué un saut qualitatif considérable.

1. Extrait de la déclaration d'Uruguay, dans J. Croome, Reshaping the World Trading System. A History of the Uruguay Round, Genève, World Trade Organization, 1995 (extrait p. 382-392). 


\section{Le GATT et la construction d'une conjoncture critique internationale}

Reproduction du scénario historique «classique » des rounds précédents, les négociations d'Uruguay se sont caractérisées par un affrontement bilatéral entre les États-Unis et la Communauté européenne, dans lequel les premiers ont adopté une attitude particulièrement offensive ${ }^{1}$, tandis que la seconde est restée dans une position essentiellement défensive. Le volet agricole est progressivement apparu comme le point d'achoppement majeur entre les protagonistes ; lors de la conférence ministérielle du Heysel (décembre 1990), initialement prévue pour clore le round, aucun accord n'a pu être trouvé sur le volet agricole alors que des compromis semblaient envisageables sur les autres volets en négociation (industrie, services). La coalition formée par les États-Unis et les pays du groupe de Cairns ${ }^{2}$ refusant de poursuivre les discussions sur les autres volets tant qu'un accord ne serait pas obtenu sur l'agriculture, la conférence s'est soldée par une grave «crise », qui menaçait directement l'existence et la poursuite du GATT.

En l'absence d'une solution sur le volet agricole, la négociation globale était directement remise en question et l'accord général sur le commerce international menacé de rupture. Mais l'ensemble des parties contractantes, y compris les Européens, avait des intérêts importants à la conclusion d'un accord global. Une réforme de la PAC, dont il est apparu progressivement qu'elle permettrait de relancer le processus de négociation, s'est ainsi imposée comme une «nécessité » tant interne qu'externe pour la Communauté européenne si elle voulait préserver et poursuivre l'échange politique international. C'est ainsi que les négociations du GATT ont fourni les conditions politiques du déclenchement d'une réforme de la PAC.

La Commission européenne a ainsi pris l'initiative politique du changement en émettant une première proposition de réforme début 1991 (projet « Mac Scharry) ${ }^{3}$ qui, outre une forte baisse des prix garantis, invitait à une compensation différentielle de cette baisse en fonction de la taille des exploitations. Sous la pression française notamment, celle-ci fut rejetée par le Conseil de l'agriculture, car, d'une part, elle entrait en contradiction avec la rhétorique de la FNSEA, puissant porte-parole professionnel national, et, d'autre part, elle aurait fortement grevé le retour budgétaire de la France sur la PAC par rapport aux autres États membres, d'autre part (ce second point valant également pour les Britanniques). La seconde proposition ${ }^{4}$, qui ne faisait plus mention de critères sociaux de modulation du soutien, a ensuite servi de base aux négociations du Conseil, qui ont finalement mené au compromis de réforme du 21 mai 1992.

1. Proposition «Option Zéro » - élimination de l'ensemble des soutiens - en juillet 1987, proposition d'avril 1989, proposition d'octobre 1989, proposition d'octobre 1990. Pour une description détaillée des négociations, cf., par exemple, J. Croome, ibid., et pour une approche plus analytique, cf. W. D. Coleman, S. Tangermann, "The 1992 CAP reform, the Uruguay Round and the Commission », Journal of Common Market Studies, 37 (3), 1999, p. 385-405. Cf. aussi H. Guyomard, L. P. Mahé, «La réforme de la PAC et les négociations du GATT, quelle compatibilité ? », Le Sélectionneur français, 45, 1995, p. 3-30.

2. Groupe de 14 pays exportateurs nets de produits agricoles, dont l'Argentine, l'Australie, la Nouvelle-Zélande, la Thaílande et l'Uruguay, qui, sans être aussi catégoriques, adoptaient des positions très proches des États-Unis, et attaquaient les mesures protectionnistes européennes.

3. Commission des Communautés européennes, « Évolution et avenir de la PAC », COM (91) 100 final, $1^{\text {er février } 1991 .}$

4. Commission des Communautés européennes, « Évolution et avenir de la Politique agricole commune, propositions de la Commission », COM (91) 258 final, 12 juillet 1991. 
Comme de nombreux travaux l'ont souligné, la volonté de «céder » aux pressions internationales est le résultat d'un arbitrage politique entre les États membres de la Communauté '. Les intérêts commerciaux en jeu sur le volet industriel du GATT et les pressions subies par le gouvernement Kohl de la part de ses milieux d'affaires dans le sens d'un dénouement rapide de la négociation expliquent que l'Allemagne ait finalement opté en faveur de la réforme. Comme nous l'avons déjà largement développé dans des travaux antérieurs ${ }^{2}$, le retournement de la position française a été tout aussi déterminant. Annoncé au dernier moment par le ministre Louis Mermaz du fait de l'opposition violente de la FNSEA, il s'explique essentiellement par la position favorable à la réforme des gros exportateurs céréaliers spécialisés français, ainsi que par l'assurance pour la France de conserver ses transferts économiques et financiers sur la PAC grâce à la compensation intégrale de la baisse des prix.

\section{ÉLITES ADMINISTRATIVES INTERNATIONALES ET PROMOTION} DES IDÉES RÉFORMISTES

\section{L'OCDE comme laboratoire d'idées international}

Comme on l'a déjà évoqué, le Trade Mandate Model et les ESP mis au point à l'OCDE ont été très largement utilisés par les différents États pour affirmer et argumenter leurs propositions et leurs positions respectives au GATT ${ }^{3}$. Mais le rôle de l'OCDE est allé bien au-delà. La conception de «l'efficacité » économique dont cette organisation était porteuse du fait de l'intensité de ses échanges avec le forum scientifique (formation de ses acteurs et mobilisation de l'expertise académique) a poussé les négociations commerciales vers des critères d'acceptabilité pour les politiques agricoles domestiques beaucoup plus restrictifs qu'ils n'auraient pu l'être a priori. Tout système de contingentement de l'offre, jugé fondamentalement inefficace car responsable de distorsions, était ainsi rejeté au profit de systèmes minimisant l'intervention directe des pouvoirs publics sur le marché. C'est ainsi que s'est imposé le critère du « découplage » des aides, notion particulièrement intéressante car elle illustre parfaitement les modalités du passage des idées d'un forum à un autre. Répondant à une définition stricte sur le forum scientifique ${ }^{4}$, elle a été importée au niveau international du forum des communautés de politiques publiques où elle fait l'objet d'une définition beaucoup plus floue et négociable : tout l'enjeu des négociations agricoles d'Uruguay a justement consisté à décider si telle ou telle aide devait ou non être considérée comme « découplée ».

Sous l'influence des acteurs de l'OCDE, la notion du découplage s'est donc progressivement imposée comme un des critères internationaux majeurs d'acceptabilité

1. Cf., par exemple, W. D. Coleman, S. Tangermann, «The 1992 CAP Reform... », art. cité. Pour un compte rendu des événements par le directeur général de l'Agriculture à la Commission européenne, cf. G. Legras, «L'Uruguay Round et la réforme de la PAC », Politique étrangère, 58, 1993.

2. Cf. Ė. Fouilleux, « Le polycentrisme : contraintes et ressources stratégiques. Le cas de la Politique agricole commune », dans J. Commaille, B. Jobert (dir.), Les métamorphoses de la régulation politique, Paris, LGDJ, 1999 (coll. « Droit et société »), et Ė. Fouilleux, « La cogestion à l'épreuve de l'Europe. L'exemple de la réforme de la Politique agricole commune », Grenoble, CERAT-CNRS, 1996.

3. C. Cahill, W. Legg, dans OCDE, « Modélisation... », art. cité.

4. En théorie, une aide découplée est une aide qui n'affecte pas la fonction de production de l'agriculteur, c'est-à-dire qui n'interfère pas dans ses décisions de production (surfaces, types de productions, etc.). 


\section{Ève Fouilleux}

des politiques domestiques. Le nombre d'alternatives envisageables pour une éventuelle réforme de la PAC s'en est ainsi trouvé particulièrement réduit. Or cela n'était pas évident au départ : initialement, les négociations visaient à débarrasser le marché mondial des distorsions commerciales, sans présumer des instruments de politique agricole à mettre en œuvre dans cette optique au niveau domestique. Le commentaire suivant d'un acteur interrogé à l'OCDE au sujet de cette période illustre bien la manière dont les élites internationales du forum des communautés de politique publique se sont emparées du débat sur la PAC :

« Il faut bien comprendre qu'ici, à l'OCDE, on regardait les bénéfices économiques globaux qui pouvaient résulter d'un accord dans le cadre de l'Uruguay Round... Il y a une chose, c'est le gain économique général qu'on peut tirer d'un paquet de mesures de libéralisation sur un ensemble de secteurs. OK. Mais il y en a une autre : ce sont les gains domestiques, en dehors des gains liés au commerce, qu'on peut en tirer... Nous, dans une perspective propre à l'OCDE, ce qu'on voulait tirer d'un accord à l'Uruguay Round, c'était d'améliorer bien sûr la situation des échanges commerciaux mais aussi de se débarrasser des inefficacités qui existaient au niveau domestique. Parce que vous savez, vous pouvez très bien résoudre... vos problèmes commerciaux avec des quotas de production par exemple, en gelant vos structures de production, avec tous ces machins-là quoi... Et là, il y avait là un grand désaccord. Notamment avec les Américains. À cette époque, les Américains disaient : on se fiche de comment ils [les Européens] le font,... tant qu'ils nous débarrassent des distorsions commerciales. Mais bien sûr, nous, à l'OCDE on ne pouvait pas être d'accord avec ça. Pour nous il fallait bien sûr qu'ils se débarrassent des distorsions commerciales, mais aussi des distorsions domestiques. C'est ça notre agenda...

Je me rappelle avoir été à Washington à la fin des années 1980 pour discuter comment la position américaine au GATT pouvait changer. Et une des choses sur laquelle on travaillait à l'époque, c'était qu'on réalisait tous qu'une réforme de la PAC était absolument essentielle pour obtenir un accord dans le cadre de l'Uruguay Round. Le problème était de savoir quelle était la réforme de la PAC qui permettrait d'obtenir un accord raisonnable au GATT. Ce que nous étions nombreux à pousser à l'époque, c'était que la Communauté devait introduire un système de paiements directs. Et en fait, c'est d'ailleurs exactement ce qu'ils ont fini par faire !" '.

Outre leur rôle dans l'importation initiale des grilles d'analyse scientifiques sur le forum des communautés de politique publique, les économistes de l'OCDE ont ainsi également été les vecteurs privilégiés de la progression de ces idées jusqu'à leur institutionnalisation finale au niveau européen à travers la réforme de la PAC et au niveau international à travers la clôture du Round d'Uruguay en 1994 ?

1. Cette citation illustre l'imbrication au quotidien des réseaux d'acteurs impliqués dans les différentes discussions internationales. Les échanges entre administrations nationales et internationales doivent aussi être mentionnés. Certains personnels du secrétariat de l'OCDE, par exemple, étaient en détachement d'administrations nationales comme l'Economic Research Service (bureau d'analyse statistique et de prospective de l'US Department of Agriculture, ministère de l'Agriculture américain) ou l'Australian Bureau of Agriculture Economics, et sont rentrés ensuite dans leur administration d'origine, pour s'occuper, entres autres, des négociations de 1'Uruguay Round.

2. L'accord de Marrakech définit plusieurs «boites » de classement des mesures de soutien en fonction de leur impact sur les échanges : dans la boîte verte sont placées les mesures sans effet notable de distorsion (qui restent donc autorisées), dans la boîte bleue figurent les mesures ayant un impact direct (soumises à une réduction échelonnée), et dans la boîte rouge les mesures interdites. Les Européens ont réussi à faire classer (transitoirement ?) les paiements compensatoires de la PAC de 1992 dans la boîte verte. 


\section{La Commission européenne, médiateur européen de l'ajustement}

Dans un sens, à travers sa proposition de réforme (aides directes découplées), le rôle de la Commission européenne a donc consisté à introduire dans la négociation intergouvernementale européenne les idées en vigueur dans le débat commercial international. Mais le rôle de la Commission ne doit pas pour autant être réduit à celui d'une stricte courroie de transmission des idées de l'OCDE. Supposer une telle «neutralité » dans la médiation négligerait un facteur important : les élites administratives européennes elles-mêmes, leurs idées et leur volonté politique. Si la Commission a longtemps fait preuve d'une certaine inertie à propos des questions agricoles, l'arrivée de l'équipe Delors à sa tête a marqué un changement radical de son discours sur la PAC : non seulement ses dysfonctionnements étaient un obstacle budgétaire majeur au développement d'autres politiques européennes, mais ils risquaient en outre de délégitimer l'entreprise européenne dans son ensemble '.

Dans le «Livre Vert sur l'avenir de la politique agricole commune » de $1985^{2}$, une réforme était ainsi présentée comme un impératif politique de premier ordre. Mais au-delà de cette «mise sur l'agenda » du changement, le Livre Vert revêtait une dimension programmatique fondamentale. Les prix garantis n'y étaient plus considérés comme des instruments de soutien des revenus (idée encore présente dans les réflexions de la Commission de 1980 , par exemple ${ }^{3}$ ), mais comme des générateurs d'inefficacités économiques. Par ailleurs, s'il était stipulé que l'agriculture ne pouvait fonctionner sans aucun soutien public, celui-ci devait néanmoins être sélectif et versé à ceux « qui en ont le plus besoin ». Au terme d'une revue commentée des instruments de politique envisageables dans la perspective d'une réforme, la baisse des prix garantis des céréales compensée par un système d'aides directes était présentée comme la seule alternative envisageable pour la PAC.

Sur le plan de la sociologie des élites administratives, l'arrivée de l'équipe Delors a marqué le début d'un processus de reconquête par les "réformistes » de la direction générale de l'Agriculture (DG VI), dont le monopole a longtemps expliqué l'inertie de la Commission au sujet de la PAC. À travers l'investissement et la revalorisation des activités de prospective en son sein, ces acteurs (qui, pour plusieurs d'entre eux, avaient reçu une formation dans le cadre du paradigme scientifique dominant en économie) ont peu à peu fait progresser leurs idées jusqu'à la rédaction secrète đu projet de réforme Mac Sharry dans les derniers mois de 1990. Alors qu'ils avaient été jusque-là contraints à une certaine timidité, compte tenu du risque de rejet de leurs propositions par le Conseil en première lecture, ces « réformistes » ont trouvé dans la conjoncture critique établie au GATT le moyen de faire passer, à la fois au sein de la Commission et auprès du Conseil, une réforme qu'ils défendaient déjà depuis plusieurs années.

La réforme de la PAC de 1992 peut ainsi être considérée comme le produit d'un ajustement global / sectoriel et interne / externe européen issu de la volonté politique des États membres de 1'Union dans lequel la Commission a joué un rôle de médiateur actif.

1. Cf. G. Ross, « Organizing European Space», dans Jacques Delors and European Integration, Oxford, Oxford University Press, 1995, p. 107-213.

2. Commission des Communautés européennes, «Perspectives pour la Politique agricole commune », «Livre Vert », (communication de la Commission au Conseil et au Parlement), COM (85) 333 final, Bruxelles, 30 juillet 1985.

3. Commission des Communautés européennes, « Réflexions sur la Politique agricole commune », (communication de la Commission au Conseil), COM (80) 800 final, Bruxelles, 5 décembre 1980 . 
UNE INTERACTION CONTINUELLE ENTRE INSTITUTIONNALISATION ET PRODUCTION DES IDÉES

\section{Ajustement paradigmatique sur le forum scientifique}

Alors que les économistes du bien-être clamaient vainement depuis l'origine leur scepticisme sur la PAC aux niveaux nationaux et européen du forum, les effets d'apprentissage induits par la connaissance scientifique ont donc finalement débouché sur un changement radical de la politique, qualifié par eux-mêmes de « grand pas dans la bonne direction »'. Comme le déclarait le président de l'Association européenne des économistes agricoles: «La mesure de la progression vers le marché dans le domaine de la politique agricole constitue un des rares domaines dans lequel les avancées scientifiques académiques ont eu un rôle prééminent sur les résultats politiques obtenus » 2 .

C'est au niveau international du forum des communautés de politique publique, à travers les travaux de l'OCDE, que les scientifiques ont été entendus en premier car les outils conceptuels qu'ils proposaient permettaient de déchiffrer et de traiter le problème posé à cet endroit du forum des communautés de politique publique. Outre leur connaissance empirique du fonctionnement des marchés et des politiques agricoles, ils disposaient des outils théoriques et des techniques de modélisation économétrique adéquates pour faire le lien entre échanges commerciaux et instruments des politiques domestiques. La forte influence des élites administratives anglo-saxonnes (nord-américaines en particulier) au sein des organisations internationales est un autre élément d'explication: structurellement beaucoup plus proches des milieux universitaires académiques ${ }^{3}$, elles sont ainsi beaucoup plus sensibles aux enjeux et évolutions du forum scientifique et susceptibles d'en mobiliser les acteurs.

En contrepoint de l'alimentation du débat politique par les idées scientifiques, le renouvellement du débat politique a eu des effets de feed-back importants sur le forum scientifique lui-même, particulièrement clairs dans le cas français. Contrairement aux autres forums nationaux, le forum français de l'économie agxicole a longtemps été dominé par le paradigme marxiste. Entièrement focalisé sur l'étude des effets du modèle modernisateur et sur les politiques des structures agricoles, les études sur les politiques de prix, dont la PAC, y ont ainsi été particulièrement sous-développées ${ }^{4}$. Le renouvellement du débat politique au début des années 1980 (réformes de la PAC, problème des marchés agricoles) a clairement permis aux tenants du paradigme néoclassique d'amorcer le retournement paradigmatique $\mathrm{du}$ forum ${ }^{5}$. Outre l'audience

1. Cf. H. Guyomard, L. P. Mahé, « La réforme de la PAC. Une révolution ou un grand pas dans la bonne direction? ", Revue du Marché commun et de l'Union européenne, 366, 1993, p. 222-236.

2. Cf. A. Buckwell, « Agricultural Economics... », cité.

3. A. de Janvry, "L'économie rurale aux États-Unis : état actuel et perspectives », Économie rurale, 200, 1990, p. 48-50.

4. Cf. sur ce point les critiques des lacunes françaises par Michael Tracy dans son article : «Les économistes et la politique agricole », Économie rurale, 200, 1990, p. 21-23. Pour une vision d'auteurs inscrits dans le paradigme marxiste, cf. M. Blanc, P. Lacombe, «Quarante ans d'économie rurale ", dans M. Bodiguel, P. Lowe, Campagne française, campagne britannique, Paris, L'Harmattan, 1989, p. 125-153 et M. Tracy, « Les économistes et la politique agricole », art. cité, p. 21-23.

5. L'organisation d'une session de la Société française d'économie rurale en mars 1983 sur le thème « Bilan de 15 ans de leadership marxiste, la remontée des réalistes » peut être considérée comme une date-clé de ce point de vue. 
nouvelle que leur a conféré le renouvellement du débat européen et international sur les politiques agricoles ', leur accès à l'expertise (au sein de l'OCDE mais également à la Commission européenne ${ }^{2}$ ) a certainement constitué une ressource importante pour prouver la validité de leur paradigme et achever leur processus traductionnel.

\section{Feed-back du changement institutionnel. Mutations des débats} sur le forum professionnel

La défense du niveau des prix garantis de la PAC, au centre des revendications du porte-parole, a longtemps occulté toute autre dimension du débat concernant la politique agricole sur le forum professionnel. À la base de compromis fondateurs internes depuis les années 1960 (dispositifs de redistribution entre céréaliers et éleveurs), les prix garantis étaient une forme de ciment de l'unité syndicale ; leur universalité d'application (« un prix est le même pour tous ») constituait par ailleurs un point d'ancrage essentiel de la rhétorique unitaire de la FNSEA. Si la période de négociation en 1991-1992 (conjoncture de crise) n'a pas contribué à clarifier le débat du fait de la violence des réactions qu'elle a engendrée chez les agriculteurs, le changement institutionnel qu'elle a induit s'en ait, semble-t-il, chargé « à retardement ».

Soulignons tout d'abord que les instruments introduits en 1992 (aides directes) ont les mêmes effets sur le revenu des différentes catégories de producteurs concernés que les précédents (prix garantis) : du fait de la compensation intégrale de la baisse des prix pour l'ensemble des producteurs, ils ont été calculés de manière à reproduire exactement la répartition antérieure du soutien (à quelques variations régionales près). En revanche, alors que le soutien octroyé par les prix garantis était particulièrement opaque, les aides directes sont calculées sur la base de la surface de l'exploitation et versées chaque année sous la forme d'un chèque au producteur. Leur intelligibilité immédiate, leur forte visibilité et leur meilleure imputabilité ont été à l'origine d'une profonde transformation des controverses sur le forum professionnel.

La soudaine visibilité des disparités induites a tout d'abord fait resurgir le débat si longtemps occulté de l'équité et de la justice sociale dans la distribution du soutien public à l' agriculture : est-il légitime que les agriculteurs les plus « gros » reçoivent le plus ? Autour du thème du «plafonnement des aides», la FNSEA a ainsi été contrainte de se départir de sa rhétorique unitaire sur l'égalité de condition du monde paysan, et de laisser publiquement apparaître les fortes divergences d'intérêt caractérisant les différentes composantes du monde agricole et rural. Par ailleurs, alors que le syndicalisme de gauche était resté très minoritaire jusqu'alors ${ }^{3}$, l'apparition de cette nouvelle dimension du débat a renforcé la légitimité de la Confédération paysanne comme porte-parole alternatif du forum, tant sur le terrain qu'auprès des pouvoirs publics. Ainsi, alors que la Commission y avait échoué en 1991 (rejet immédiat de son premier texte), ce sont les agriculteurs eux-mêmes qui, quelques années plus tard, ont relancé la dimension « sociale » du débat sur la politique agricole.

1. Pour une argumentation dans ce sens, cf. D. Vermersch, Économie politique agricole et morale sociale de l'Église, Paris, Economica, 1997.

2. Cf. le rapport d'expertise «Dysharmonies in EC and US Agricultural Policy Measures » (publié par la Commission en 1988).

3. Malgré la création de la Confédération paysanne en 1987 suite au rassemblement progressif des gauches syndicales, sa représentativité est restée faible dans les années 1980 . Cf. les résultats aux élections professionnelles présentés par Bertrand Hervieu dans : I. Boussard, D. Boy, J. Chiche, B. Hervieu, N. Mayer, "Attitudes politiques des agriculteurs», Paris, FNSP-CNRS, 1995 (Les Cahiers du CEVIPOF. 12). 
Corrélée à l'accumulation de problèmes liés aux excès de l'agriculture intensive et du modèle moderniste (pollution des eaux par les pesticides et nitrates d'origine agricole, vache folle, scandales sanitaires divers), la nouvelle visibilité du soutien a également contribué à l'addition d'une dimension environnementale au débat sur la politique agricole. Autrefois totalement absente, cette dimension est notamment posée en ces termes : dans un contexte de « verdissement " généralisé des discours, est-il normal que les agriculteurs les plus intensifs, souvent les moins respectueux de l'environnement et les plus éloignés d'un modèle d'agriculture « durable », soient ceux qui touchent le plus d'aides publiques ' ? Des propositions en termes de « conditionnalité environnementale » du soutien (octroi des aides sur critères de «bonnes pratiques », par exemple) font ainsi aujourd'hui partie intégrante du débat sur la PAC, chose impensable il y a encore quelques années.

Ce nouveau contexte a non seulement permis l'incursion d'acteurs extérieurs dans le débat agricole comme les ONG d'environnement, mais il a également induit une redistribution des cartes à l'intérieur du forum en termes de modèle légitime d'agriculture : la visibilité plus grande conférée aux groupes d'agriculteurs promoteurs de modèles alternatifs (agriculture biologique, agriculture durable) leur a effectivement permis de relancer leurs processus traductionnels et de renforcer leurs dispositifs d'intéressement ${ }^{2}$. Ils sont de plus en plus souvent consultés par les pouvoirs publics nationaux (ministère de l'Environnement, mais également ministère de l'Agriculture) et européens (DG VI, DG XI).

Sans doute l'apparition de ces deux «nouvelles » dimensions du débat sur le forum professionnel (équité et justice sociale, environnement et développement durable) a-t-elle par ailleurs préparé le terrain pour des mobilisations de plus grande ampleur. Les militants, issus du milieu agricole « alternatif » dissident par rapport à la FNSEA, ont ainsi formé une grande partie des bataillons qui se sont rendus à Seattle fin 1999 pour s'opposer au lancement du « cycle du Millénaire » par l'Organisation mondiale du commerce, bataillons auxquels ils ont également fourni, en la personne de José Bové, un porte-parole actif et médiatique.

\section{ESPACES ET TEMPS DE LA SÉLECTION DES IDÉES}

La construction d'un cadre analytique formalisé pour analyser l'articulation entre idées et institutions nous a permis de souligner que la sélection des alternatives de politique publique est un processus progressif, complexe et interactif. Sur chaque forum, il commence à la source de la production des idées à travers la définition d'un référent central dominant spécifique et d'un porte-parole légitime. La dynamique des

1. Le débat concernant la «prime au maïs ensilage » (aliment du bétail dans les formes d'élevage intensif) est exemplaire de ce point de vue : suite à la réforme de 1992, les mesures « agri-environnementales » accordent une « prime à l'herbe » de l'ordre de 350 francs par hectare aux éleveurs « extensifs » pour encourager une agriculture plus respectueuse de l'environnement (animaux en pâture). Mais que dire de cette incitation quand une «prime au maïs ensilage » de l'ordre de 2500 francs par hectare est octroyée au titre des paiements compensatoires?

2. Si les écrits d'André Pochon, par exemple, figure emblématique de l'agriculture durable en Bretagne (cf. A. Pochon, Du champ à la source, op. cit.), sont restés inconnus du grand public jusqu'au milieu des années 1990, il est de plus en plus souvent sollicité par les grands médias nationaux aujourd'hui, et son dernier ouvrage a été publié par un éditeur national (cf. André Pochon, Les champs du possible, Paris, La Découverte - Syros, 1998). 
idées s'explique ensuite par les enjeux de légitimation qui animent les acteurs qui les portent et par les échanges politiques qu'ils établissent entre les différents forums. Elle se termine lors de la fabrication des instruments de politique publique, forme d'institutionnalisation des idées. À l'interface entre les producteurs d'idées et les hommes politiques élus, les élites administratives ont un rôle essentiel dans l'importation des idées et dans leur transformation en recettes applicables de politique publique.

Du point de vue empirique, l'application de ce cadre d'analyse a permis de mettre en évidence les étapes successives qui ont mené à la réforme de la PAC de 1992. Tout d'abord, un renouvellement important des connaissances et des techniques sur le forum scientifique a induit une évolution de la problématisation des politiques agricoles par les économistes spécialistes du champ. L'échange qu'a établi l'OCDE avec ces derniers au début des années 1980 a ensuite donné naissance à de nouveaux outils légitimes pour analyser les politiques agricoles, outils au contenu normatif particulièrement important. Puis, les conditions politiques d'institutionnalisation de ces nouvelles idées se sont successivement établies au niveau international (ouverture d'un volet agricole dans le cadre de l'Uruguay Round du GATT), puis au niveau européen (comportement d'entrepreneur politique par la Commission, volonté des États membres de poursuivre de l'échange politique international, renouvellement du compromis franco-allemand sur la PAC).

L'association de la notion de forum à une approche de la politique publique comme ensemble d'institutions endogènes nous a ensuite permis d'illustrer l'étendue possible des répercussions d'un changement de politique sur le plan cognitif. Deux exemples ont été développés sur ce point. Le premier concerne le renouvellement du débat et des demandes d'expertise sur la PAC au cours des années 1980, qui a fourni des ressources importantes aux économistes du bien-être pour affirmer la pertinence de leur paradigme sur le forum scientifique français de l'économie rurale. Le second a décrit le bouleversement du débat professionnel du fait de la visibilité et de l'imputabilité accrue des instruments de la Nouvelle PAC par rapport aux précédents, qui s'est traduit par des changements progressifs dans la hiérarchie des modèles d'agriculture et des porte-parole légitimes sur le forum.

Enfin, l'analyse des étapes successives de l'institutionnalisation des idées réformistes sur la PAC aux niveaux européen et international du forum a clairement souligné l'existence de lieux et de temps différents pour l'élaboration des alternatives de politique publique et pour leur négociation. À cette distinction tant spatiale que temporelle correspondent tout d'abord des acteurs différents : contrairement aux États membres qui interviennent en phase de négociation, la Commission et les services du secrétariat général de l'OCDE disposent des ressources sur le moyen terme pour mettre au point instruments d'analyse, indicateurs et autres critères divers d'efficacité ou de bonne gouvernance, qui sont autant d'arguments destinés à alimenter les négociations et délibérations à venir. Le cas empirique étudié a montré l'enjeu stratégique et politique que constitue l'organisation de la prospective et de l'expertise au niveau international. Dans la perspective d'une affirmation de l'Union européenne comme entité politique autonome sur la scène internationale, la structuration institutionnelle des ressources intellectuelles disponibles devrait donc être considérée comme une priorité. Si la Commission tente apparemment de réorganiser ses services dans ce sens, reste à savoir si elle doit être la seule institution dépositaire de cette tâche au niveau européen.

À cette distinction correspond également un degré de publicité différent des débats, l'opacité et la dimension normative des travaux menés entre experts sur les 
politiques agricoles n'étant contrebalancée aux niveaux européen et international par aucun dispositif institutionnel permettant un échange politique direct entre les instances dépositaires du pouvoir politique et la société civile. Si les quelques mobilisations transnationales apparues ces dernières années autour de questions européennes ou globales peuvent donner l'impression de combler en partie ce déficit ', elles restent sporadiques, incertaines et leurs tenants ne disposent pas de réelles ressources dans un échange qui reste uniquement cantonné à la conjoncture critique. L'élaboration de dispositifs institutionnalisés permettant un échange politique réel entre les parties prenantes à ces niveaux sera sans doute un défi majeur à relever, en particulier au sein de l'Union européenne si l'on veut en faire une composite polity ${ }^{2}$ plus... démocratique ".

Ève Fouilleux est Chargée de recherche au Centre de recherches administratives et politiques (Rennes), actuellement Jean Monnet Fellow au Centre Robert Schuman de l'Institut universitaire européen (Florence). Elle a soutenu sa thèse de science politique «Idées, institutions et dynamiques du changement de politique publique. Les transformations de la Politique agricole commune », sous la direction de Bruno Jobert, à l'université Pierre Mendès France de Grenoble, le 29 janvier 1999. Elle a publié «Changement de politique publique dans l'Union européenne : la Politique agricole commune entre permanences et innovations ", Politiques et management public, 15 (1), mars 1997, p. 117-137 ; « Le polycentrisme : contrainte et ressource stratégique. Le cas de la Politique agricole commune », dans Jacques Commaille, Bruno Jobert, Les métamorphoses de la régulation politique, Paris, LGDJ, 1999 (coll. « Droit et société »), p. 177-202, et va publier Idées et changement institutionnel. Les réformes de la Politique agricole commune, Paris, L'Harmattan (à paraître en 2000). Ses thèmes de recherche portent actuellement sur le rôle des grandes organisations internationales dans la production des normes et recettes de politique publique, sur les modalités de l'introduction de critères de respect de l'environnement et de développement durable dans les politiques économiques et sur les enjeux de la comitologie au sein du système politique européen (CRAP, IEP de Rennes, 104 Boulevard de la Duchesse Anne, 35700 Rennes).

\section{RÉSUMÉ/ABSTRACT}

ENTRE PRODUCTION ET INSTITUTIONNALISATION DES IDÉES. LA RÉFORME DE LA POLITIQUE AGRICOLE COMMUNE

La grille d'analyse proposée dans cet article a pour objectif de comprendre l'articulation entre les phénomènes de production et d'institutionnalisation des idées. Elle associe une approche institutionnaliste des instruments de politique publique au concept de forum, opérationnalisé à travers la sociologie de la traduction et la notion d'échange politique. La partie empirique étudie les processus qui ont mené à la réforme de la Politique agricole commune de 1992. Elle analyse la production par l'OCDE de nouveaux critères de bonne politique agricole domes-

1. Cf. D. Imig, S. Tarrow (eds), Contentious Europeans..., op. cit.

2. Ibid.

* En grande partie tiré de ma thèse de doctorat, cet article a été rédigé dans le cadre d'un séjour postdoctoral (Jean Monnet Fellowship) au Centre Robert Schuman de l'Institut universitaire européen de Florence. 


\section{La réforme de la Politique agricole commune}

tique dans les années 1980 à partir d'idées issues du forum scientifique des économistes, puis leur introduction par la Commission dans la négociation intergouvernementale européenne. Le rôle d'encadrement de la production des idées par les instruments de politique publique est ensuite souligné à travers l'évolution des débats scientifique et professionnel comme conséquence du changement de 1992.

BETWEEN PRODUCTION AND INSTITUTIONALIZATION OF IDEAS. REFORMING THE [EU'S] COMMON AGRICULTURAL POLICY

The purpose of the analysis framework suggested in this article is to understand the mechanisms at work in the production and the institutionalization of ideas. It associates the institutionalist approach to public policy instruments and the concept of a forum, operationalized through sociology of translation and the concept of political exchange. The empirical part studies the processes that led to the 1992 reform of the CAP. It analyses the production by OECD of new criteria of good domestic agricultural policy in the 1980s, based on ideas discussed in the economists' scientific forum, and then their introduction by the Commission in the European intergovernmental negotiation. The framing role performed by public policy instruments vis-à-vis the production of ideas is then underlined by reviewing the changing scholarly and professional debates, as a consequence of the 1992 reform. 
